\title{
Formação de Grupos de alunos baseada em Múltiplos Critérios
}

\author{
Giuseppe F. Neto ${ }^{1}$, Lucas A. Santos ${ }^{1}$, Samuel B. J. Souza ${ }^{2}$, Mayara S. O. Castro ${ }^{1}$, \\ Lizandra L. S. B. Silva ${ }^{1}$, Rafael F. L. Mello ${ }^{1}$, Péricles B. C. Miranda ${ }^{1}$ \\ ${ }^{1}$ Departamento de Estatística e Informática \\ Universidade Federal Rural de Pernambuco (UFRPE) \\ ${ }^{2}$ Centro de Informática \\ Universidade Federal de Pernambuco (UFPE)
}

\begin{abstract}
Group formation is one of the main steps of the collaborative learning. This paper proposes an intelligent method to optimize the group formation process considering multiple criteria: inter-homogeneity, intra-heterogeneity and empathy. The method was evaluated regarding the performance, being compared to the exhaustive and random approaches; And regarding the pedagogical aspect, being compared with random and self-selected methods. The results showed the potential of the proposed method from the computational point of view as well as the pedagogical point of view.
\end{abstract}

Resumo. A formação de grupos é uma das etapas mais importantes da aprendizagem colaborativa. Este artigo propõe um método inteligente para otimizar o processo de formação de grupos levando em conta múltiplos critérios: inter-homogeneidade, intra-heterogeneidade e empatia. $O$ método foi avaliado quanto ao desempenho, sendo comparado às abordagens exaustiva e aleatória; e quanto ao aspecto pedagógico, sendo comparado com os métodos aleatório e auto-selecionado. Os resultados obtidos mostraram o potencial do método proposto tanto do ponto de vista computacional quanto do pedagógico.

\section{Introdução}

A aprendizagem colaborativa (AC) é um modelo baseado na coesão de trabalhos individuais em determinado grupo, através de discussão e construção conjunta do conhecimento [Dillenbourg 1999]. Embora muitos estudos demonstrem a utilidade da AC no contexto educacional [Nielsen et al. 2009], um número reduzido trata sobre uma questão importante: o procedimento a ser utilizado para a formação dos grupos. O processo de formação de grupos não é trivial, devido à variedade de fatores (histórico de notas de um aluno, características pessoais e habilidades) e combinações existentes à medida que o número de alunos aumenta [Liu and Tsai 2008]. Uma má formação de grupos pode afetar a realização dos objetivos estabelecidos para o grupo, prejudicando a aprendizagem dos membros [Dillenbourg 1999].

Este trabalho propõe um método evolucionário para formação de grupos, considerando um número arbitrário de características dos indivíduos. Nesta pesquisa, a tarefa de formação de grupos é tratada como um problema de otimização com múltiplos critérios em um cenário combinatório. Foram considerados três objetivos: 1) maximizar 
a inter-homogeneidade, propondo maior igualdade entre grupos distintos; 2) maximizar a intra-heterogeneidade, promovendo a diversidade dos alunos (alunos com característica e habilidades distintas) dentro de cada grupo; 3 ) maximizar a empatia, de modo que membros pertencentes a um grupo tenha afinidade entre si. O método proposto foi avaliado sobre o ponto de vista de desempenho e o pedagógico. Do ponto de vista de desempenho, comparamos o método com as abordagens exaustiva e aleatória, usando um conjunto de dados de valores simulados (uniformemente distribuídos). Do ponto de vista pedagógico, o mesmo foi avaliado através de um experimento real, com estudantes do primeiro ano do curso de programação de computadores. O método foi comparado com duas estratégias tradicionais de formação de grupos: aleatória e auto-organizada (por empatia). Os resultados mostraram que, tanto do ponto de vista de desempenho quanto do pedagógico, o método proposto é eficiente e capaz de formar grupos adequados, levando-se em conta os três objetivos considerados.

Este artigo está organizado da seguinte forma: a Seção 2 introduz o contexto do problema de formação de grupos e os principais trabalhos relacionados. A Seção 3 descreve detalhadamente o método desenvolvido. A Seção 4 apresenta a metodologia experimental utilizada para avaliar o método proposto. A Seção 5 apresenta os resultados alcançados. Finalmente, a Seção 6 destaca as conclusões e trabalhos futuros.

\section{Formação de Grupos}

A formação de grupos tem sido investigada por décadas em áreas como psicologia, sociologia e educação [Owens et al. 1998]. Estas áreas contribuíram bastante quanto ao entendimento das relações sociais entre indivíduos e como as habilidades de cada um podem favorecer o crescimento dos outros e do grupo. No entanto, vale salientar a grande contribuição da área de computação na automatização desta atividade, reduzindo assim o tempo gasto necessário à sua conclusão.

A formação de grupos pode ser categorizada quanto à forma, abordagens e/ou critérios. A forma está relacionada à maneira como a formação do grupo é feita, podendo o grupo ser definido manualmente pelo professor, ou de forma automática através de algum sistema. Em relação às abordagens, existem três importantes: a aleatória, que forma grupos aleatoriamente; A auto-selecionada, onde os próprios membros definem o grupo em que desejam ficar; E a selecionada, que é quando um sistema determina os critérios para a formação de grupos. Outra forma de categorização é quanto aos critérios. Os mais destacados na literatura são: o homogêneo, quando cada grupo adota estudantes de características similares para a formação de grupos; o heterogêneo, quando cada grupo adota estudantes de características diferentes para a formação de grupos; E ambos, quando o grupo for misto, isto é, adotar estudantes homogêneos em alguma(s) característica(s) e heterogêneos em outra(s), ou quando permite formar tanto grupos homogêneos quanto grupos heterogêneos. De acordo com [Monteverde et al. 2016], a grande utilidade de métodos computacionais na formação de grupos é comprovada, uma vez que grande parte dos trabalhos encontrados na literatura indicam que a forma mais utilizada é a automática e que a abordagem mais comum é a selecionada por sistema.

Em relação aos métodos computacionais já propostos na literatura, destacam-se aqueles que utilizam abordagens inteligentes [Pedrycz et al. 2016]. Estes métodos, além de automatizar a tarefa de formação de grupos, tentam encontrar soluções de forma efi- 
VI Congresso Brasileiro de Informática na Educação (CBIE 2017)

Anais do XXVIII Simpósio Brasileiro de Informática na Educação (SBIE 2017)

ciente e eficaz. O trabalho desenvolvido por [Graf and Bekele 2006] usa uma abordagem de otimização de enxame de partículas (PSO) e considera os níveis e interesses de compreensão dos alunos. O trabalho desenvolvido por [Contreras et al. 2016] adota um algoritmo genético (GA) aprimorado e considera o número de conceitos já conhecidos de um determinado domínio do curso e as notas de pré-testes. Já o trabalho de [Lin et al. 2010] usa um método de colônia de formigas (ACO) e considera o desempenho geral do aluno e uma estimativa de seus traços de personalidade. O trabalho desenvolvido por [Pinheiro et al. 2014] adotou um algoritmo de agrupamento para identificação de grupos de alunos baseado no log em ambientes virtuais de aprendizagem. Além dos trabalhos mencionados previamente, também destaca-se o proposto por [Ani et al. 2010], que apresenta um método para formação de grupo usando GA para a formação de grupos de desenvolvimento de software.

Embora os trabalhos anteriores sejam relevantes, os mesmos apresentam duas limitações. A primeira delas é quanto ao número de características consideradas, no máximo três características para descrever os indivíduos. A segunda é quanto ao número de objetivos levados em consideração na otimização dos grupos. Apenas um único critério é considerado (Homogeneidade ou Heterogeneidade). No entanto, a formação de grupos é um problema com vários objetivos/critérios que necessitam ser satisfeitos em conjunto. Diferentemente dos estudos apresentados, propomos um método para a formação automática de grupos considerando múltiplos critérios: intra-heterogeneidade, inter-homogeneidade e empatia entre membros dos grupos; e, além disso, uma gama diversa de características para a descrição dos membros. Vale salientar que este trabalho é pioneiro, ao considerar um cenário multi-objetivo.

\section{Trabalho Desenvolvido}

O método proposto para a formação de grupos é descrito nesta seção. O problema em questão demanda um espaço de soluções de dimensão elevada, com múltiplos objetivos a serem levados em conta. Diante disto, decidimos utilizar um algoritmo genético multiobjetivo (MOGA).

\subsection{Otimização Multi-Objetivo}

A otimização multi-objetivo (MO) objetiva buscar por soluções que satisfaçam mais de um objetivo ao mesmo tempo. Considere um vetor $\vec{C}=\left(C_{1}, C_{2}, \ldots C_{k}\right)$ de critérios, onde $k$ é o número de objetivos a serem considerados na otimização. Deste modo, cada solução possui um vetor $\vec{f}=\left(f_{1}, f_{2}, \ldots, f_{k}\right)$, onde cada valor (fitness) de $\vec{f}$ representa a avaliação da solução em cada critério pertencente a $\vec{C}$. Como neste trabalho são adotados três objetivos na otimização $\vec{C}=\left(C_{1}, C_{2}, C_{3}\right)$, cada solução possui um vetor $\vec{f}=\left(f_{1}, f_{2}, f_{3}\right)$. Como cada solução possui um vetor de fitness, a comparação entre diferentes soluções se dá da seguinte forma. Dadas duas soluções distintas $S_{1}$ e $S_{2}$, onde $f_{\left(S_{1}\right)}$ representa o vetor de fitness de $S_{1}$ e $f_{\left(S_{2}\right)}$ representa o vetor de fitness de $S_{2} ; S_{1}$ domina $S_{2}$ se $f_{\left(S_{1}\right)}$ é melhor que $f_{\left(S_{2}\right)}$ em ao menos um fitness, e não é pior em nenhum outro. Deste modo, existem três casos possíveis: 1) $S_{1}$ domina $S_{2}, 2$ ) $S_{1}$ é dominada por $S_{2}$, ou 3) $S_{1}$ é incomparável em relação à $S_{2}$. Este último caso ocorre, quando $S_{1}$ e $S_{2}$ vencem em pelo menos um critério. O conjunto de soluções incomparáveis (ou não-dominadas) é conhecido como Pareto Front. Deste modo, algoritmos de otimização multi-objetivo tentam encontrar o Pareto Front ótimo, ou seja, aquele conjunto de soluções não-dominadas que melhor satisfazem 
os critérios considerados. No MOGA, uma população inicial de indivíduos (soluções) é definida, e esta passa, iterativamente, por processos evolucionários de avaliação, seleção, cruzamento e mutação. Após um determinado número de evoluções, o Pareto front resultante, que reflete as melhores soluções, é gerado como saída do algoritmo.

\subsection{Representação do Indivíduo}

Os indivíduos (cromossomos), representam as possíveis soluções para o problema a ser tratado. Cada cromossomo pode ser representado como um vetor de genes $\vec{I}=\left(I_{1}, I_{2}, \ldots, I_{k}\right)$, onde $k$ é o número de variáveis de decisão. No contexto do problema de formação de grupos, objetiva-se definir a melhor combinação de alunos em grupos. Para isso, determinamos que cada gene seria representado por um estudante, sendo o cromossomo um vetor de estudantes (turma), sem repetição. Como o cromossomo representa uma turma com $k$ alunos, precisando-se formar $g$ grupos, sendo $g<k$ e $k$ divisível por $g$, o vetor de estudantes $\vec{I}$ deve ser seccionado em $k / g$ grupos. O exemplo a seguir considera um possível cromossomo com $k=12$ e $g=3$ e mostra como seria a representação de cada grupo no cromossomo em questão.

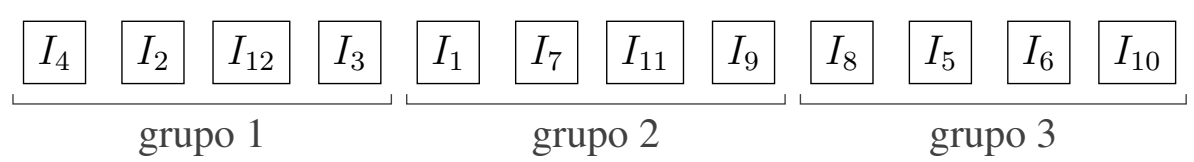

Como se pode ver no exemplo, o cromossomo possui uma combinação de 12 alunos, onde $I_{1}$ representa o aluno $_{1}, I_{2}$ representa o aluno $_{2}$, e assim sucessivamente. Os primeiros 4 genes dizem respeito aos alunos do primeiro grupo; em seguida, os próximos 4 genes dizem respeito ao segundo grupo; e finalmente, os genes restantes fazem parte do terceiro grupo.

Neste trabalho, objetiva-se otimizar a formação de grupos levando-se em consideração as características de cada estudante. Para isso, cada estudante possui um vetor de características $\vec{C}=\left(C_{1}, C_{2}, \ldots C_{n}\right)$, onde $n$ é o número de características usadas descrever o perfil do aluno. Além disso, cada estudante também possui um vetor de empatia $\left.\vec{E}=\left(E_{1}, E_{2}, \ldots, E_{(} k-1\right)\right)$ que armazena as taxas de empatia de um dado aluno em relação a cada um dos demais. Logo, se existem $k$ estudantes no cromossomo, cada estudante terá um vetor $\vec{E}$ com $k-1$ taxas de empatia. É importante mencionar que os valores contidos em $\vec{C}$ e $\vec{E}$ foram definidos no intervalo entre [0,1], onde 0 significa ruim e 1 bom.

\subsection{Avaliação do Indivíduo}

Uma das etapas mais importantes do MOGA é a avaliação dos cromossomos da população. Esta etapa determina o quão boa é a solução, e se esta será considerada na próxima geração. Neste trabalho, três objetivos foram considerados na avaliação das soluções: 1) a inter-homogeneidade, que deve ser maximizada, para garantir que os grupos sejam parecidos entre si; 2) a intra-heterogeneidade, que deve ser maximizada, para garantir que, internamente, cada grupo seja composto por estudantes de perfis diferentes; e a empatia, que deve ser maximizada, para garantir que os componentes do mesmo grupo tenham afinidade e colaborem. Os cálculos necessários para a avaliação das soluções serão apresentados a seguir. 
VI Congresso Brasileiro de Informática na Educação (CBIE 2017)

Anais do XXVIII Simpósio Brasileiro de Informática na Educação (SBIE 2017)

Cálculo da Inter-homogeneidade (Inter Hom): O cálculo da inter-homogeneidade entre os grupos é dividido em alguns passos. O primeiro é a criação de uma matriz $M A C$ de alunos $\times$ características, sendo os valores das características de cada aluno normalizadas entre [0,1]. $M A C$ é utilizada para calcular o vetor $V T=\left(\overline{C_{1}}, \overline{C_{2}}, \ldots, \overline{C_{n}}\right)$, onde $\overline{C_{i}}$ é a média dos valores da característica $i$ dentre todos os alunos.

O próximo passo é calcular a média dos valores de cada característica, mas entre os estudantes do mesmo grupo. Deste modo, cada grupo possui um vetor $\overrightarrow{V I}_{j}=$ $\left(\overline{X_{j, 1}}, \overline{X_{j, 2}}, \ldots, \overline{X_{j, n}}\right)$, onde $\overline{X_{j, i}}$ é a média dos valores da característica $i$ do grupo corrente $j$. Finalmente, o valor da inter-homogeneidade entre os grupos Inter Hom é calculado da seguinte forma:

$$
\text { Inter Hom }=\sum_{j=1}^{g}\left[\left(\overline{C_{1}}-\overline{X_{j, 1}}\right)^{2}+\left(\overline{C_{2}}-\overline{X_{j, 2}}\right)^{2}+\ldots+\left(\overline{C_{n}}-\overline{X_{j, n}}\right)^{2}\right] \text {. }
$$

Como o cálculo da inter-homogeneidade trata-se da soma do quadrado das diferenças entre $V T$ e $V I$, isso significa que quanto menor o valor de Inter Hom, mais similar cada grupo é em relação aos demais grupos. Deste modo, para maximizar a interhomogeneidade, deve-se reduzir ao máximo o valor de Inter Hom.

Cálculo da Intra-heterogeneidade (IntraHet): Para o cálculo da intraheterogeneidade, também fez-se uso da matriz $M A C$, usada no cálculo do valor de Inter Hom. Neste caso, $M A C$ é usada para definir uma matriz $M D$ aluno $\times$ aluno, que armazena os valores das distâncias de cada aluno em relação aos demais. A distância entre dois alunos $a$ e $b$ é calculada a partir da equação da distância Euclideana, levando-se em consideração as características de cada um:

$$
D_{a, b}=\sqrt[2]{\left(C_{a, 1}-C_{b, 1}\right)+\left(C_{a, 2}-C_{b, 2}\right)+\ldots+\left(C_{a, n}-C_{b, n}\right)} .
$$

Como o objetivo é maximizar a intra-heterogeneidade de cada grupo, faz-se necessário definir um valor $\overline{D G_{j}}$, que é a média das distâncias de cada estudante de um grupo $j$ em relação aos colegas da mesma equipe. $\mathrm{O}$ valor da intra-heterogeneidade total é obtido, portanto, através da média das distâncias de cada grupo (ver equação 3). Diante disto, para maximizar a intra-heterogeneidade, quanto maior o valor de IntraHet, melhor.

$$
\text { IntraHet }=\frac{\sum_{j=1}^{g} D G_{j}}{g} .
$$

Cálculo da Empatia ( $E m p$ ): Como mencionado na seção 3.2, cada estudante possui um vetor $\vec{E}$ com taxas de empatia em relação aos demais alunos. Para o cálculo de $E m p$, é necessário, inicialmente, calcular a empatia entre os alunos do mesmo grupo $\left(E G_{j}\right)$, onde $j$ é um dado grupo. O valor de $E G_{j}$ nada mais é do que a média das empatias de cada aluno em $j$, em relação aos demais também contidos em $j$. Uma vez calculada a empatia de cada grupo, Emp é a média das empatias de cada grupo (ver equação 4). Diante disto, para maximizar a empatia total, quanto maior o valor de Emp, melhor. 


$$
E m p=\frac{\sum_{j=1}^{g} E G_{j}}{g} .
$$

\section{Metodologia Experimental}

Neste trabalho, o método proposto foi analisado em dois aspectos: desempenho (quantitativo) e pedagógico. Para avaliar o desempenho do método proposto, uma comparação foi feita usando um conjunto de dados de valores simulados (uniformemente distribuídos) levando-se em conta as características descritas na seção 4.1. Nesta análise, o método foi comparado com as abordagens exaustiva e aleatória (ambas em suas versões multiobjetivo, considerando-se a inter-homogeneidade, intra-heterogeneidade e empatia). Por questões práticas de comparação dos resultados, foi decidido que, ao invés de comparar os Paretos resultantes encontrados por cada método, seria selecionada apenas uma solução de cada Pareto Front. Esta prática é muito comum em análises de métodos multi-objetivo, e geralmente utiliza-se o método de Borda Count [Orouskhani et al. 2017]. Este método utiliza uma estratégia de ranqueamento por objetivo, de modo que a solução do Pareto Front que estiver melhor ranqueada é selecionada. O propósito desta análise é constatar se o método proposto é capaz de encontrar bons grupos em tempo hábil.

Para a realização da análise pedagógica, foi realizado um experimento com estudantes do primeiro ano do curso de programação de computadores. Uma amostra de 60 alunos de três turmas diferentes (20 alunos por turma) foi selecionada para a pesquisa. Cada turma foi dividida em 5 grupos de 4 alunos. Vale salientar que o número de 4 alunos por grupo foi definido pelos professores de cada turma, uma vez que a complexidade do projeto é proporcional a este número de alunos. A atividade de aprendizagem sugerida consistiu em cada grupo de cada turma desenvolver um projeto de software (combinado entre os professores das turmas) que envolve assuntos vistos na disciplina, com um prazo de duas semanas. É importante mencionar que embora os projetos de cada grupo apresentem mesmo nível de complexidade e requisitos similares, cada equipe de cada turma é responsável por um projeto diferente para evitar cópia.

Uma vez apresentado o estudo de caso, foi realizada a comparação do método proposto com outros dois métodos tradicionais de formação de grupos: aleatória e autoselecionada. Neste experimento, cada método foi aplicado exclusivamente sobre uma turma diferente, e os grupos sugeridos por cada abordagem foram avaliados pedagogicamente, ou seja, em relação aos seus desempenhos (notas) obtidos em seus projetos. A intenção desta análise investigar se, na prática, o método proposto contribui (do ponto de vista pedagógico) com a formação de grupos que possam obter melhores desempenhos (notas) quando comparados aos das demais abordagens. O cálculo da nota final do grupo é a média entre a nota do projeto, e a média das notas de cada aluno do grupo. Deste modo, projetos que não foram entregues por completo e/ou alunos que não tenham contribuído com a realização do projeto, sejam motivo para penalização da nota do grupo.

\subsection{Características Consideradas}

Neste trabalho foram adotadas características (para descrição dos estudantes) com base em uma revisão da literatura e as características particulares da população estudada, ou seja, alunos de primeiro ano: 1) gênero, 2) habilidade em codificação, 3) habilidade em interpretação de problemas, 4) habilidade comunicativa, e 5) habilidade de liderança. Vale 
salientar que as características citadas acima são utilizadas para o cálculo de dois objetivos: a inter-homogeneidade e a intra-heterogeneidade. O terceiro objetivo, a maximização da empatia, é calculado a partir da estimativa de empatia de um aluno em relação aos demais (sexta característica), que será explicada posteriormente.

A extração das características 1 a 5 se deu através da aplicação de um questionário. O questionário consistiu em um conjunto de perguntas de resposta única e de múltipla escolha sobre tópicos relacionados à questões pessoais e técnicas. Os valores de cada uma das característica foi normalizado em um intervalo de $[0,1]$, dando uma medida quantitativa aproximada sobre quão "bom"(próximo de 1) cada aluno está em relação a cada característica. Vale salientar que a característica gênero adotou os valores 1 para feminino e 0 para masculino. Para a extração da estimativa de empatia (característica 6), utilizamos o fórum de discussões e as mensagens trocadas entre os alunos na plataforma online utilizada pelos professores para a gestão da disciplina. Deste modo, cada aluno possui um valor entre 0 (nenhuma empatia) e 1 (muita empatia) relacionado a cada um dos demais alunos de sua turma, ou seja, se a turma possui 20 alunos, cada aluno tem 19 estimativas de empatia, uma para cada membro da turma.

\subsection{Configurações}

O algoritmo de otimização multi-objetivo adotado neste trabalho é uma adaptação do Non-Dominated Sorting Genetic Algorithm (NSGA II). O método utilizou a seleção baseada em roleta onde $40 \%$ dos indivíduos da população são clonados e mantidos na geração posterior; E os demais indivíduos são considerados na etapa de cruzamento. O operador de mutação foi aplicado na população em cada geração com uma probabilidade de mutação para cada indivíduo $\theta=0.2$ e uma probabilidade de mutação para cada cromossomo $\theta=0.15$. O tamanho populacional, $T A M=50$, bem como o número máximo de gerações, $n_{-} g e n=500$, foram definidos empiricamente. O método aleatório adotou o mesmo número de soluções candidatas que as utilizadas pela proposta. No caso do método exaustivo, o número de soluções analisadas não é fixo, pois as possibilidades de formação de grupos aumenta em uma proporção fatorial de acordo com o número de estudantes. Assim, com 10 alunos, o número de soluções analisadas é $3.6 \times 10^{6}$; Com 20 alunos, esse número aumenta para $2.43 \times 10^{18}$. Os métodos aleatório e proposto foram executados 30 vezes para gerar um desempenho médio. Todos os métodos foram implementados em Python usando o ambiente Microsoft Visual Studio 2010 e todas as execuções foram realizadas em um computador laptop com um processador Intel Core i7-5600U com 4M Cache, 8GB RAM e $3.20 \mathrm{GHz}$.

\section{Resultados}

Nesta seção, os resultados são apresentados levando-se em conta duas análises diferentes. A primeira avalia o desempenho do método proposto, e a segunda investiga a sua contribuição pedagógica.

\subsection{Análise de Desempenho}

Nesta análise, comparamos o desempenho do método proposto com a abordagem exaustiva e aleatória. A Tabela 1 apresenta o tempo médio (medido em segundos) e o valor de fitness médio atingindo em cada critério (inter-homogeneidade, intra-heterogeneidade e empatia). Por uma questão de simplicidade, o número de membros por grupo foi de 5 
VI Congresso Brasileiro de Informática na Educação (CBIE 2017)

Anais do XXVIII Simpósio Brasileiro de Informática na Educação (SBIE 2017)

em todos os casos. Além disso, o desempenho de cada método foi avaliado variando-se o tamanho da turma e, consequentemente, o número de grupos.

Tabela 1. Análise comparativa do desempenho entre as abordagens exaustiva, aleatória e proposta.

\begin{tabular}{|c|c|c|c|c|c|c|c|c|c|c|c|c|}
\hline \multirow[t]{2}{*}{ "Alunos } & \multicolumn{4}{|l|}{ Exaustivo } & \multicolumn{4}{|l|}{ Aleatório } & \multicolumn{4}{|l|}{ Proposta } \\
\hline & Tempo & InterHom & IntraHet & Emp & Tempo & InterHom & IntraHet & Emp & Tempo & InterHom & IntraHet & Emp \\
\hline 10 & $\begin{array}{r}1 \\
0.46\end{array}$ & 0.21 & 0.72 & 0.71 & $\begin{array}{r}1 \\
0.52\end{array}$ & $0.35(\downarrow)$ & 0.72 & 0.69 & $\begin{array}{r}1 \\
0.56\end{array}$ & 0.21 & 0.72 & 0.71 \\
\hline 15 & 101.43 & 0.19 & 0.79 & 0.73 & 5.67 & $0.39(\downarrow)$ & $0.52(\downarrow)$ & $0.52(\downarrow)$ & 7.98 & 0.22 & 0.74 & 0.67 \\
\hline 20 & 987.51 & 0.13 & 0.80 & 0.80 & 10.76 & $0.42(\downarrow)$ & $0.49(\downarrow)$ & $0.47(\downarrow)$ & 15.34 & 0.17 & 0.74 & 0.73 \\
\hline 25 & 8843.2 & 0.09 & 0.85 & 0.84 & 30.45 & $0.48(\downarrow)$ & $0.44(\downarrow)$ & $0.40(\downarrow)$ & 43.23 & 0.13 & 0.79 & 0.78 \\
\hline 30 & 16560.4 & 0.04 & 0.88 & 0.88 & 60.12 & $0.50(\downarrow)$ & $0.39(\downarrow)$ & $0.39(\downarrow)$ & 80.67 & $0.13(\downarrow)$ & 0.81 & $0.78(\downarrow)$ \\
\hline 50 & N/A & N/A & N/A & N/A & 180.89 & 0.54 & 0.33 & 0.28 & 200.56 & 0.13 & 0.81 & 0.79 \\
\hline 100 & N/A & N/A & N/A & N/A & 400.23 & 0.64 & 0.22 & 0.22 & 450.98 & 0.12 & 0.80 & 0.79 \\
\hline
\end{tabular}

Como esperado, o tempo necessário para o método exaustivo avaliar todas as possibilidades aumenta dramaticamente à medida que o número de estudantes também aumenta. Vale salientar que, para 50 e 100 alunos, o método exaustivo tornou-se impraticável, e por estas razões, o resultado não foi apresentado. Em contrapartida, o tempo de execução do método proposto e aleatório aumenta quase que linearmente. Também pode-se observar que o método exaustivo obtém valores de fitness melhores à medida que o número de estudantes aumenta, porque, com mais alunos, mais combinações de grupos podem ser formadas. O mesmo comportamento não acontece com o método aleatório, pois, à medida que o número de soluções possíveis aumenta, as chances de encontrar boas formações de grupos aleatoriamente diminuem.

É importante destacar que o método proposto, mesmo não atingindo valores ótimos (exatamente iguais aos atingidos pelo exaustivo) em todos os cenários, foi capaz de obter boas soluções em tempo de execução menor, até quando o número de alunos é grande. Com o intuito de obter comprovação estatística, o teste de hipótese $t$-student foi aplicado, considerando $95 \%$ de significância. Os valores de fitness, apresentados na Tabela 1, que estiverem em negrito significam que são estatisticamente iguais aos do exaustivo. Por outro lado, os valores com o símbolo $\downarrow$ são estatisticamente inferiores aos do exaustivo. A comparação estatística realizada não levou em consideração os valores para 50 e 100 alunos, uma vez que os resultados do método exaustivo não foram obtidos por questões de custo computacional.

Como se pode ver, a maioria dos resultados alcançados pelo método aleatório, para cada objetivo, foram superados estatisticamente pelos resultados da abordagem exaustiva. Apenas no cenário mais simples que a abordagem aleatória teve bons resultados. Por outro lado, os valores de fitness obtidos pelo método proposto foram estatisticamente iguais aos do método exaustivo nos cenários de 10, 15, 20 e 25 alunos. Embora os valores para 30 unidades tenham sido estatisticamente piores que o do exaustivo, os resultados foram bons e alcançados em bem menos tempo. Talvez, com mais gerações, melhores resultados teriam sido obtidos. Com relação aos resultados para 50 e 100 alunos, o método proposto foi capaz de melhorar levemente os valores de fitness dos três objetivos. Quando comparado à abordagem aleatória, o método proposto a superou estatisticamente em todos os objetivos e na maioria dos cenários. 
VI Congresso Brasileiro de Informática na Educação (CBIE 2017)

Anais do XXVIII Simpósio Brasileiro de Informática na Educação (SBIE 2017)

\subsection{Análise Pedagógica}

O desempenho pedagógico dos grupos formados leva em consideração a nota do projeto. O cálculo da nota do grupo é a composição da nota do projeto e de cada membro do grupo (como explicado na seção 4). A Tabela 2 apresenta os resultados pedagógicos colhidos através da aplicação dos grupos formados pelas três abordagens: proposta, aleatória e auto-selecionada, em turmas de 20 estudantes (5 grupos de 4 alunos).

São apresentados na Tabela 2, por abordagem de agrupamento, a média e desvio padrão das notas dos projetos de cada turma; a média e o desvio padrão das notas dos indivíduos de cada turma; e a média e o desvio padrão das notas dos grupos de cada turma. Os professores relataram que os projetos desenvolvidos por equipes formadas a partir do método proposto conseguiram entregar o projeto completo. A média das notas dos projetos entregues é 8.6 com um desvio padrão de 1.4. Em relação às notas individuais, percebeu-se também a contribuição efetiva de cada componente de cada equipe em seus projetos. Como consequência, a nota média dos grupos formados pela proposta foi bastante satisfatória.

Tabela 2. Média das notas dos grupos e individuais de cada abordagem.

\begin{tabular}{ccccc}
\hline \hline & N. Alunos & Notas dos Projetos & Notas Individuais & Notas dos Grupos \\
\hline Proposta & 20 & $8.6( \pm 1.4)$ & $8.8( \pm 0.98)$ & $8.7( \pm 1.19)$ \\
Aleatória & 20 & $5.4( \pm 2.34)$ & $6.2( \pm 0.42)$ & $5.8( \pm 1.38)$ \\
Auto-selecionada & 20 & $6.4( \pm 3.0)$ & $6.6( \pm 2.9)$ & $6.5( \pm 2.9)$ \\
\hline
\end{tabular}

Em relação à abordagem auto-selecionada, pode-se ver que a média das notas nos projetos foi 6.4 , mas com 3.0 de desvio padrão, ou seja, as notas dos projetos das equipes variaram bastante. $\mathrm{O}$ resultado das notas individuais seguiram o mesmo comportamento, a média foi de 6.6 e o desvio padrão de 2.9. Esta informação confirma que a contribuição dos alunos foi desbalanceada, ou seja, alguns alunos não colaboraram como deveriam, enquanto outros ficaram sobrecarregados. Por este motivo, alguns projetos, das equipes que adotaram a abordagem auto-selecionada, não foram entregues por completo. A média do grupo, portanto, foi penalizada, resultando em 6.5. Quanto à abordagem aleatória, os resultados mostraram que esta não foi capaz de formar boas equipes. Como pode-se ver, a média das notas individuais dos membros dos grupos formados aleatoriamente é 6.2 , com baixo desvio padrão, ou seja, as notas dos alunos em grupos aleatórios não variam muito, ficando próximas da média. O que torna crítica a situação dos grupos formados aleatoriamente é a nota do projeto, 5.4. De acordo com o relato dos professores, algumas equipes com esta formação não chegaram a entregar todos os requisitos do projeto. Diante de tudo isto, a nota média das equipes com esta formação é 5.8 .

\section{Conclusão e Trabalhos Futuros}

Este trabalho propõe um método automático para otimizar a formação de grupos levando em consideração múltiplos critérios: a inter-homogeneidade, intra-heterogeneidade e empatia. Os resultados mostraram que o método proposto alcançou desempenho similar estatisticamente ao do método exaustivo na maioria dos cenários, mas com custo de tempo linear. Além disso, o método também foi comparado com uma abordagem aleatória de formação de grupos, superando-a em todos os critérios. Quanto à análise pedagógica, foi constatado que os grupos formados a partir do método proposto obtiveram melhores 
VI Congresso Brasileiro de Informática na Educação (CBIE 2017)

Anais do XXVIII Simpósio Brasileiro de Informática na Educação (SBIE 2017)

notas na atividade dada quando comparados com os grupos formados a partir dos demais métodos. Futuramente, pretende-se aplicar o método proposto em turmas de Massive Open Online Courses (MOOC) com um número maior de alunos. Além disso, objetivamos levar em consideração mais informações a respeito dos alunos para melhor definir o perfil de cada um.

\section{Referências}

Ani, Z. C., Yasin, A., Husin, M. Z., and Hamid, Z. A. (2010). A method for group formation using genetic algorithm. International Journal on Computer Science and Engineering, 2(9):30603064.

Contreras, R., Salcedo, P., et al. (2016). Genetic algorithms as a tool for structuring collaborative groups. Natural Computing, pages 1-9.

Dillenbourg, P. (1999). Collaborative learning: Cognitive and computational approaches. advances in learning and instruction series. ERIC.

Graf, S. and Bekele, R. (2006). Forming heterogeneous groups for intelligent collaborative learning systems with ant colony optimization. In International Conference on Intelligent Tutoring Systems, pages 217-226. Springer.

Lin, Y.-T., Huang, Y.-M., and Cheng, S.-C. (2010). An automatic group composition system for composing collaborative learning groups using enhanced particle swarm optimization. Computers \& Education, 55(4):1483-1493.

Liu, C.-C. and Tsai, C.-C. (2008). An analysis of peer interaction patterns as discoursed by on-line small group problem-solving activity. Computers \& Education, 50(3):627-639.

Monteverde, I., Ramos, D., Gadelha, B., Oliveira, E., and do Nascimento, P. (2016). Formação de grupos em ambientes virtuais de aprendizagem: Uma revisão sistemática da literatura. In Brazilian Symposium on Computers in Education (Simpósio Brasileiro de Informática na EducaçãoSBIE), volume 27, page 310 .

Nielsen, T., Hvas, A. E., and Kjaergaard, A. (2009). Student team formation based on learning styles at university start: does it make a difference to the student. Reflection Education, 5(2):85103.

Orouskhani, M., Teshnehlab, M., and Nekoui, M. A. (2017). Evolutionary dynamic multiobjective optimization algorithm based on borda count method. International Journal of Machine Learning and Cybernetics, pages 1-29.

Owens, D. A., Mannix, E. A., and Neale, M. A. (1998). Strategic formation of groups: Issues in task performance and team member selection. Research on managing groups and teams, $1: 149-165$.

Pedrycz, W., Sillitti, A., and Succi, G. (2016). Computational intelligence: an introduction. In Computational Intelligence and Quantitative Software Engineering, pages 13-31. Springer.

Pinheiro, M. F., Neto, L. C. F., de Sá Junior, H. N., da Mata, E. C., Jacob Jr, A. F., and de Lima Santana, Á. (2014). Identificação de grupos de alunos em ambiente virtual de aprendizagem: Uma estratégia de análise de log baseada em clusterização. In Anais dos Workshops do Congresso Brasileiro de Informática na Educação, volume 3, page 582. 